\title{
Spectrum of Kelvin-wave turbulence in superfluids
}

\author{
Victor S. L'vov* and Sergey Nazarenko ${ }^{\dagger}$ \\ * Department of Chemical Physics, The Weizmann Institute of Science, Rehovot 76100, Israel, \\ $\dagger$ Mathematics Institute, Warwick University, Coventry, CV4 7AL, UK
}

(Dated: August 2, 2021)

\begin{abstract}
We derive a type of kinetic equation for Kelvin waves on quantized vortex filaments with random large-scale curvature, that describes step-by-step (local) energy cascade over scales caused by 4 -wave interactions. Resulting new energy spectrum $E_{\mathrm{LN}}(k) \propto k^{-5 / 3}$ must replace in future theory (e.g. in finding the quantum turbulence decay rate) the previously used spectrum $E_{\mathrm{KS}}(k) \propto k^{-7 / 5}$, which was recently shown to be inconsistent due to nonlocality of the 6 -wave energy cascade.
\end{abstract}

1. Introduction. Nowadays, turbulence in superfluids $[1,2]$ is attracting more and more attention, stimulated by advances in experimental techniques allowing studies of turbulence in various systems such as ${ }^{3} \mathrm{He}$, $[3,4],{ }^{4} \mathrm{He}[5,6]$ and Bose-Einstein condensates of supercold atoms $[7,8]$, and also by an impressive progress in numerical simulations $[9,10]$ which give access to characteristics of turbulence yet unavailable experimentally. One of the most interesting questions is the nature of the energy dissipation, observed in turbulence of inherently dissipation-free superfluids. This is especially intriguing in zero-temperature limit, when normal fluid component disappear together with the obvious dissipation mechanisms: viscosity of normal fluids and mutual friction between the superfluid and the normal components.

Superfluid turbulence (ST) comprises a tangle of quantized vortex lines which can be characterized by the intervortex distance $\ell$ and the vortex core radius $a$. At scales $L \gg \ell$ the discreteness is unimportant and they can be described classically with the energy flux toward smaller scales by the Richardson-Kolmogorov cascade.

Then the energy is transferred through the crossover scale $\ell$ by some complicated mechanisms $[12,16]$, thereby exciting smaller scales $\ell<\lambda<a$ which propagate along the individual vortex filaments as waves. These were predicted by Kelvin more than one century ago [11] and experimentally observed in superfluid ${ }^{4} \mathrm{He}$ about 50 years ago. It is believed that Kelvin waves (KWs) play a crucial role in superfluid dynamics, transferring energy from $\ell$ to a much smaller scale, where it can dissipate via emission of bulk phonos. In a wide range of scales KWs are weakly nonlinear and can be treated within the theory of weakwave turbulence [15]. Such an approach for KWs was initiated and developed in [12] where a 6 -wave kinetic equation (KE) was presented, and a $\mathrm{KW}$ spectrum (8) was obtained from this equation based on a dimensional analysis. This spectrum was subsequently used in theoretical constructions in ST, e.g. to describe the classicalquantum crossover range of scales and to explain the ST dissipation rate $[12,16]$. However, it was recently shown in [13] that spectrum (8) is nonlocal and, therefore, nonrealizable. This crucial locality check was only possible after a highly non-trivial calculation of the 6-wave interaction coefficient done in Refs. [16] which took into account previously omitted important contributions.
In this Letter, we exploit the consequences of the nonlocality of the 6 -wave theory, and replace the latter with a new local 4-wave theory of KW turbulence. Such a 4wave theory arises from the full 6-wave theory (completed in [16]) in the strongly nonlocal case, when two waves in the sextet re much longer than the other 4 . These waves correspond to the outer scale, - infrared (IR) cutoff. We derive a new spectrum of the KW turbulence which is local, and which must be used in future for revising the parts of the ST where the nonlocal spectrum of the 6wave theory has previously been used.

2. General background on 4-wave and 6-wave weak turbulence. We begin with a brief overview of weak-wave turbulence for the 4 -wave (of $3 \leftrightarrow 1$ type) and the 6 -wave systems, because these types will be relevant for our subsequent discussion. Let us start with a classical Hamiltonian equation for the complex canonical amplitude of waves $a_{\boldsymbol{k}} \equiv a(\boldsymbol{k}, t)$ and $a_{\boldsymbol{k}}^{*}$ (classical analogues of the Bose creations and annihilation operators) with a wavevector $\boldsymbol{k}$ :

$$
i \frac{\partial a_{k}}{\partial t}=\frac{\delta \mathcal{H}}{\delta a_{k}^{*}} .
$$

Here $\mathcal{H}$ is a Hamiltonian,

$$
\mathcal{H}=\mathcal{H}_{\text {free }}+\mathcal{H}_{\text {int }} ; \quad \mathcal{H}_{\text {free }}=\int \omega_{k} a_{\boldsymbol{k}} a_{\boldsymbol{k}}^{*} d \boldsymbol{k},
$$

where $\omega_{k}$ is the wave frequency. For KWs $\omega_{k}=\Lambda \kappa k^{2} / 4 \pi$ where $\Lambda=\ln (\ell / a)$ and $\kappa$ is the circulation quantum. $\mathcal{H}_{\text {int }}$ is an effective interaction Hamiltonian equal to

$$
\begin{aligned}
& \mathcal{H}_{1 \leftrightarrow 3}=\frac{1}{6} \int d \boldsymbol{k}_{1} \ldots d \boldsymbol{k}_{4} \delta_{1}^{2,3,4}\left[V_{1}^{2,3,4} a_{1} a_{2}^{*} a_{3}^{*} a_{4}^{*}+\text { c.c. }\right], \\
& \mathcal{H}_{3 \leftrightarrow 3}=\frac{1}{36} \int d \boldsymbol{k}_{1} \ldots d \boldsymbol{k}_{6} \delta_{1,2,3}^{4,5,6} W_{1,2,3}^{4,5,6} a_{1} a_{2} a_{3} a_{4}^{*} a_{5}^{*} a_{6}^{*},
\end{aligned}
$$

for the 4 -wave $(1 \leftrightarrow 3)$ and the 6 -wave $(3 \leftrightarrow 3)$ systems respectively. Here we use shorthand notations: $a_{j} \equiv a_{\boldsymbol{k}_{j}}$ and $\delta_{1,2,3}^{4,5,6} \equiv \delta\left(\boldsymbol{k}_{1}+\boldsymbol{k}_{2}+\boldsymbol{k}_{3}-\boldsymbol{k}_{4}-\boldsymbol{k}_{5}-\boldsymbol{k}_{6}\right), k_{j}=\left|\boldsymbol{k}_{j}\right|$.

Statistical description of weakly interacting waves can be reached [15] in terms of the $\mathrm{KE}$

$$
\frac{\partial n(\boldsymbol{k}, t)}{\partial t}=\operatorname{St}(\boldsymbol{k}, t),
$$


for the waveaction spectrum $n(\boldsymbol{k}, t)$, defined by $\left\langle a(\boldsymbol{k}, t) a^{*}\left(\boldsymbol{k}^{\prime}, t\right)\right\rangle=n(\boldsymbol{k}, t) \delta\left(\boldsymbol{k}-\boldsymbol{k}^{\prime}\right)$, where $\langle\ldots\rangle$ stands for the ensemble averaging. The collision integral $\operatorname{St}(\boldsymbol{k}, t)$ can be found in various ways [15], including the Golden Rule widely used in quantum mechanics. For the 4 -wave $(1 \leftrightarrow 3)$ and the 6 -wave $(3 \leftrightarrow 3)$ we have respectively

$$
\begin{aligned}
& \mathrm{St}_{1 \leftrightarrow 3}=\frac{\pi}{12} \int d \boldsymbol{k}_{1} \ldots d \boldsymbol{k}_{3}\left\{\left|V_{\boldsymbol{k}}^{1,2,3}\right|^{2} \delta_{\boldsymbol{k}}^{1,2,3} \mathcal{N}_{\boldsymbol{k}}^{1,2,3}\right. \\
& \quad \times \delta\left(\omega_{\boldsymbol{k}}-\omega_{1}-\omega_{2}-\omega_{3}\right) \\
& \left.+3\left|V_{1}^{\boldsymbol{k}, 2,3}\right|^{2} \delta_{1}^{\boldsymbol{k}, 2,3} \mathcal{N}_{1}^{\boldsymbol{k}, 2,3} \delta\left(\omega_{1}-\omega_{\boldsymbol{k}}-\omega_{2}-\omega_{3}\right)\right\} \\
& \mathcal{N}_{1}^{2,3,4} \equiv n_{1} n_{2} n_{3} n_{4}\left(n_{1}^{-1}-n_{2}^{-1}-n_{3}^{-1}-n_{4}^{-1}\right) \\
& \mathrm{St}_{3 \leftrightarrow 3}=\frac{\pi}{24} \int d \boldsymbol{k}_{1} \ldots d \boldsymbol{k}_{5}\left|W_{\boldsymbol{k}, 1,3}^{4,5,6}\right|^{2} \delta_{\boldsymbol{k}, 1,3}^{4,5,6} \\
& \quad \times \delta\left(\omega_{\boldsymbol{k}}+\omega_{1}+\omega_{2}-\omega_{3}-\omega_{4}-\omega_{5}\right) n_{\boldsymbol{k}} n_{1} n_{2} n_{3} n_{4} n_{5} \\
& \quad \times\left(n_{1}^{-1}+n_{2}^{-1}+n_{3}^{-1}-n_{4}^{-1}-n_{5}^{-1}-n_{6}^{-1}\right) .
\end{aligned}
$$

Scaling solutions of these KE's (up to a constant prefactor $A$ ),

$$
n(k)=A k^{-x},
$$

can be found under two conditions [15]:

- Scale-invariance of the wave system, when the frequency of waves and the interaction coefficients are homogeneous functions of wave vectors: $\omega(\lambda k)=$ $\lambda^{\alpha_{2}} \omega(k), V\left(\lambda \boldsymbol{k}_{1} ; \lambda \boldsymbol{k}_{2}, \lambda \boldsymbol{k}_{3}, \lambda \boldsymbol{k}_{4}\right) \equiv \lambda^{\alpha_{4}} V\left(\boldsymbol{k}_{1} ; \boldsymbol{k}_{2}, \boldsymbol{k}_{3}, \boldsymbol{k}_{4}\right)$, and a similar relationship for $W_{1,2,3}^{4,5,6}$ with an index $\alpha_{6}$.

- Interaction locality, in a sense that the main contribution to the energy balance of a given $k$-wave (with wavevector $k$ ) originates from its interaction with $k^{\prime}$ waves with $k^{\prime} \sim k$. Mathematically it means that all integrals over $k_{1}, k_{2}$, etc. in the KE's (4) converge, and therefore the leading contribution to the collision integral indeed originates from the regions $k_{2} \sim k, k_{3} \sim k$, etc. Note that nonlocal spectra are not solutions of the KE's (4) and, therefore, physically irrelevant.

To find the scaling index $x$ for turbulent spectra with a constant energy flux over scales, we note that all KE's (4) conserve the total energy of the wave system, $d E / d t=0, \quad E \equiv \int E_{\boldsymbol{k}} d \boldsymbol{k}, \quad E_{\boldsymbol{k}} \equiv \omega_{\boldsymbol{k}} n_{\boldsymbol{k}}$. Therefore the $\boldsymbol{k}$-space energy density, $E_{\boldsymbol{k}}$, satisfies a continuity equation: $\frac{\partial E_{k}}{\partial t}+\frac{\partial \varepsilon_{k}}{\partial k}=0$. Here $\varepsilon_{k}$ is the energy flux over scales, expressed via an integral over sphere of radius $k$ : $\varepsilon_{k}=\int_{k^{\prime}<k} d \boldsymbol{k}^{\prime} \omega_{k^{\prime}} \operatorname{St}\left(k^{\prime}, t\right)$.

Under the assumption of the interaction locality, one estimates the $d$-dimensional integral $\int d \boldsymbol{k}$ as $k^{d}$, the interaction coefficients $V_{1}^{2,3,4} \sim V_{k}^{k, k, k} \sim V k^{\alpha_{4}}, W_{1,2,3}^{4,5,6} \sim$ $W_{k, k, k}^{k, k, k} \sim W k^{\alpha_{6}}$ and $n_{k}=A_{p} k^{-x_{p}}$ (for the $p$-wave interactions). Therefore:

$$
\begin{aligned}
& \varepsilon_{k} \sim k^{3 d}\left(V k^{\alpha_{4}}\right)^{2}\left(A_{4} k^{-x_{4}}\right)^{3}, 1 \Leftrightarrow 3 \text { scatering; (6a) } \\
& \varepsilon_{k} \sim k^{5 d}\left(W k^{\alpha_{6}}\right)^{2}\left(A_{6} k^{-x_{6}}\right)^{5}, 3 \Leftrightarrow 3 \text { scattering. (6b) }
\end{aligned}
$$

For the spectra of turbulence with a constant energy flux $\varepsilon_{k}=\varepsilon=$ const., i.e. $\varepsilon_{k} \propto k^{0}$. For the $p$-wave process this gives the scaling exponent of $n(k), x_{p}$, and the energy scaling exponent $y_{p}, E(k) \propto k^{-y_{p}}$ :

$$
x_{p}=d+2 \alpha_{p} /(p-1), \quad y_{p}=x_{p}-\alpha_{2} .
$$

In fact, these expressions are valid for any $p>2$. For the 3 - and the 4 -wave processes (with $p=3$ and $p=$ 4) this gives the well-known results, see e.g. Ref. [15]. Note however, that the 4 -wave $1 \leftrightarrow 3$ is considered here for the first time, and it is different from the previously considered standard $2 \leftrightarrow 2$ processes.

3. 6-wave $K \boldsymbol{W}$ turbulence. Finding the effective interaction Hamiltonian $\mathcal{H}_{\text {int }}$ for KWs appears to be a hard task. For the 6-wave process, which assumes that the underlying vortex is perfectly straight, this task was accomplished only recently Refs. [16]. Effective $3 \leftrightarrow 3$ interaction coefficient $W$ was shown to have a form

$$
W_{1,2,3}^{4,5,6}=-3 \boldsymbol{k}_{1} \boldsymbol{k}_{2} \boldsymbol{k}_{3} \boldsymbol{k}_{4} \boldsymbol{k}_{5} \boldsymbol{k}_{6} F_{1,2,3}^{4,5,6} / 4 \pi \kappa,
$$

where $F$ is a non-singular dimensionless function of $\boldsymbol{k}_{1}, \ldots \boldsymbol{k}_{6}$, close to unity in the relevant region of its arguments (KW case is $1 \mathrm{D}$, but we still use boldface for the wavevectors, reserving non-bold notation as $k_{j}=\left|\boldsymbol{k}_{j}\right|$ ). Notice that the form of Eq. (7) could be expected because it demonstrates a very simple physical fact: long KWs (with small $k$ 's) can contribute to the energy of a vortex line only when they produce curvature. The curvature, in turn, is proportional to wave amplitude $a_{k}$ and, at fixed amplitude, is inversely proportional to their wave-length, i.e. $\propto k$. Therefore in the effective motion equation each $a_{j}$ has to be accompanied by $k_{j}$, if $k_{j} \ll k$. Exactly this statement is reflected by Eq. (7). One can say that cumbersome calculations [13] support these reasoning, and additionally provide with an explicit expression for $F$.

Equation (7) estimates $W_{1,2,3}^{4,5}$ as $W k^{6}$. Thus, Eq. (6b) reproduces the Kozik-Svistunov (KS) scaling for the $3 \leftrightarrow 3$ processes, which for further discussion is writhen with a dimensionless constant $C_{\mathrm{KS}}$ :

$$
n_{\mathrm{KS}}=\frac{C_{\mathrm{KS}} \kappa^{2 / 5} \varepsilon^{1 / 5}}{k^{17 / 5}} \Rightarrow E_{\mathrm{KS}}=\frac{C_{\mathrm{KS}} \Lambda \kappa^{7 / 5} \varepsilon^{1 / 5}}{k^{7 / 5}},
$$

Nonlocal $(3 \leftrightarrow 3)$ Kozik-Svistunov (KS) spectrum.

4. Nonlocality of the 6 -wave $K \boldsymbol{W}$ theory. To test locality of the KS spectrum (8), let us consider the $3 \leftrightarrow 3$ collision term (4c) for $\mathrm{KW}$ with the interaction amplitude $\mathcal{W}_{1,2,3}^{4,5,6}$ as in (7) and $n(k)$ as in Eq. (5). In the IR region $k_{1} \ll k, k_{j}, j=2,3,4,5$, we have $F \rightarrow 1$ and the integral over $\boldsymbol{k}_{1}$ scales as:

$$
\Psi \equiv \frac{2}{\kappa} \int_{1 / \ell} k_{1}^{2} n\left(k_{1}\right) d k_{1}=\frac{2 A}{\kappa} \int_{1 / \ell} k_{1}^{2-x} d k_{1} .
$$

Lower limit 0 in Eq. (9) is replaced by $1 / \ell$, where $\ell$ is the mean inter-vortex separation $\ell$, at which approximation of non-interacting vortex lines fails and one expects 
a cutoff of the power like behavior (5). Prefactor 2 in Eq. (9) reflects the fact that the ranges of positive and negative $\boldsymbol{k}_{1}$ give equal contributions, and factor $1 / \kappa$ is introduced to make parameter $\Psi$ dimensionless. $\Psi$ has a meaning of the mean-square angle of the deviation of the vortex lines from straight. Therefore $\Psi \lesssim 1$; for highly polarized vortex lines $\Psi \ll 1$.

Clearly, integral (9) IR-diverges if $x>3$, which is the case for the KS spectrum (8) with $x_{6}=17 / 5$. Note that all the similar integrals over $\boldsymbol{k}_{2}, \boldsymbol{k}_{3}, \boldsymbol{k}_{4}$, and $\boldsymbol{k}_{5}$ in Eq. (4c) also diverge exactly in the same manner as integral (9). Moreover, when two of the wavenumbers belonging to the same side in the sextet tend to zero simultaneously then each of such wavenumbers will yield an integral as in (9), and the net result will be the product of these integrals, i.e. a stronger singularity than in the case of just one small wavenumber. On the other hand, small wavenumbers which are on the opposite sides of the resonant sextet do not lead to a stronger divergence because of an extra smallness arising in this case in (4c) from $\left(n_{1}^{-1}+n_{2}^{-1}+n_{3}^{-1}-n_{4}^{-1}-n_{5}^{-1}-n_{6}^{-1}\right)$.

Divergence of the integrals in Eq. (4c) means that KSspectrum (8) is not a solution of the KE (4c) and thus non-realizable. One should find another, self-consistent solution of this $\mathrm{KE}$.

5. Effective 4-wave theory. Thus, the strongest nonlocality of the 6 -wave theory arises from those sextets that contain, on the same sextet side, two small wavenumbers with $k_{j} \lesssim 1 / \ell$. Thus the 6 -wave resonance conditions $\omega_{k}+\omega_{1}+\omega_{2}=\omega_{3}+\omega_{4}+\omega_{5}, \boldsymbol{k}+\boldsymbol{k}_{1}+\boldsymbol{k}_{2}=$ $\boldsymbol{k}_{3}+\boldsymbol{k}_{4}+\boldsymbol{k}_{5}$, effectively become

$$
\begin{array}{ll}
\boldsymbol{k}=\boldsymbol{k}_{1}+\boldsymbol{k}_{2}+\boldsymbol{k}_{3}, & \boldsymbol{k}_{1}=\boldsymbol{k}+\boldsymbol{k}_{2}+\boldsymbol{k}_{3}, \\
\boldsymbol{k}_{2}=\boldsymbol{k}+\boldsymbol{k}_{1}+\boldsymbol{k}_{3}, & \text { or } \quad \boldsymbol{k}_{3}=\boldsymbol{k}+\boldsymbol{k}_{2}+\boldsymbol{k}_{1},
\end{array}
$$

and respective conditions for the frequencies, which implies a 4 -wave process of the $(1 \leftrightarrow 3)$-type. In the other words, one can interpret such nonlocal sextets on straight vortex lines as quartets on curved vortices, with the slowest modes in the sextet responsible for the large-scale curvature $R$ of the underlying vortex line in the 4 -wave approach.

To derive an effective 4-wave KE, let us start with the 6 -wave collision integral (4c) and find the leading contributions to it when the spectrum $n_{k}$ is steeper than $k^{-3}$ in the IR-region. There are four of them. The first one originates from the region where $k_{1}$ and $k_{2}$ are much smaller than the rest of $k_{j}$ 's. The three other contributions originate from the other side of the sextet: regions where either $k_{3}$ and $k_{4}$, or $k_{3}$ and $k_{5}$, or $k_{4}$ and $k_{5}$ are small. These contributions are equal and we may find only one of them and multiply the result by three. Notably, the sum of the four contributions can be written exactly in the form of the $(1 \leftrightarrow 3)$-collision term (4b) with the effective $(1 \leftrightarrow 3)$-interaction amplitude

$$
V_{1}^{2,3,4}=-3 \Psi \boldsymbol{k}_{1} \boldsymbol{k}_{2} \boldsymbol{k}_{3} \boldsymbol{k}_{4} /(4 \pi \sqrt{2}),
$$

because, as shown in [13], $\lim _{k_{1} \rightarrow 0} F\left(\boldsymbol{k}_{1}, \boldsymbol{k}_{2}, \boldsymbol{k}_{3} \mid \boldsymbol{k}_{4} \boldsymbol{k}_{5}, \boldsymbol{k}_{6}\right)=$
1. Deriving Eqs. (4b) with $V_{1}^{2,3,4}$, Eq. (11), we took only leading contributions in the respective IR regions, factorized the integrals over these wave vectors like in Eq. (9) and took only the zeroth order terms with respect to the small wavevectors (by putting these wavenumbers to zero) in the rest of the expression (4c).

Equation (4b) with $V_{1}^{2,3,4}$ as in Eq. (11) is an effective 4-wave KE, which we were aiming to obtain. This KE corresponds to interacting quartets of KWs propagating along a vortex line having a random large-scale curvature $R \lesssim \ell$. Equation (11) estimates $V_{1}^{2,3,4}$ as $\mathcal{V} k^{4}$ with $\mathcal{V} \sim$ $\Psi$. Using this scaling in Eq. (6a), we arrive at a spectrum for the $1 \leftrightarrow 3$ processes with scaling exponents $x_{4}=11 / 3$ and $y_{5}=5 / 3$,

$$
n_{\mathrm{LN}}=\frac{C_{\mathrm{LN}} \varepsilon^{1 / 3}}{\Psi^{2 / 3} k^{11 / 3}} \Rightarrow E_{\mathrm{LN}}=\frac{C_{\mathrm{LN}} \Lambda \kappa \varepsilon^{1 / 3}}{\Psi^{2 / 3} k^{5 / 3}}
$$

Local $(1 \leftrightarrow 3)$ L'vov-Nazarenko (LN) spectrum.

\section{Locality of the 4-wave LN spectrum.}

Clearly, for the new spectrum (12) to be a valid solution, it must satisfy the locality test. Thus let us substitute this spectrum into the $1 \leftrightarrow 3$ collision integral (4b) and check it convergence. Leaving details of the locality test to the online supplement to this Letter, we just outline here the main steps. Taken separately, the first and the second terms in the curly bracket yield IR divergent integrals, but when taken together the leading order singularities of these terms cancel with one another, and the net result is a IR convergent integral. In the ultraviolet (UV) region, the singularity should only be checked in the second term of the curly bracket of (4b), because the first term of this bracket does not have a UV region due to the $\omega \delta$-function. Also because of this $\delta$-function, in the UV range of the second term two of the $\boldsymbol{k}$ 's, say $\boldsymbol{k}_{1}$ and $\boldsymbol{k}_{2}$, must be large simultaneously so that $\boldsymbol{k}_{1} \simeq \boldsymbol{k}_{2}$, which leads to a UV convergent integral. Thus, the LN spectrum (12) appears to be local and, therefore, it is a valid solution for describing the $\mathrm{KW}$ turbulence.

\section{Conclusions.}

- We presented a new effective 4-wave theory of KW turbulence consisting of wave quartets interacting on vortex lines with random large-scale curvature. We derived an effective 4-wave KE, (4b), (11), and solved it to obtain a new KW spectrum (12). We proved that this spectrum is local, and therefore it is a valid solution of the $\mathrm{KE}$, which should replace the nonlocal (and therefore invalid) KS-spectrum (8) in the theory of quantum turbulence. In particular, it is now necessary to revise the theory of the classical-quantum crossover scales and its predictions for the turbulence dissipation rate $[12,16]$. Further, a similar revision is needed for the analysis of laboratory experiments and numerical simulations of superfluid turbulence, which have been done over the last five years with reliance on the un-physical KS spectrum (8).

- The difference between the LN-exponent $-5 / 3$ (see (12)) from the KS-exponent $-7 / 5$ (see (8)) is $4 / 15$ which 
is rather small. This may explain why the previous numerical experiments seem to agree with the KS spectrum, obtained numerically in [14]. However, by inspection one can also see that these results also agree with the LN slope. Differences in physical processes corresponding to the $\mathrm{KS}$ and LN spectra, result in different dimensional prefactors in these spectra, in particular the different dependence on the energy flux $\varepsilon$, as well as an extra dependence on the large-scale behavior (through $\Psi$ ) in (12). Careful examination of such prefactors is necessary in future numerical simulations in order to resolve uncertainties related to close spectrum exponents and thereby test the predicted dependencies. Such numerical simulations can be done efficiently with the Local Nonlinear Equation (LNE) suggested in [13] based on the detailed analysis of the nonlinear $\mathrm{KW}$ interactions:

$$
\begin{array}{r}
i \frac{\partial \widetilde{w}}{\partial t}+\frac{\kappa}{4 \pi} \frac{\partial}{\partial z}\left[\left(\Lambda-\frac{1}{4}\left|\frac{\partial \widetilde{w}}{\partial z}\right|^{4}\right) \frac{\partial \widetilde{w}}{\partial z}\right]=0 . \\
\text { LN equation for KWs. }
\end{array}
$$

The LNE model is similar but not identical to the Truncated LIA model of [14] (these models become asymptotically identical for weak $\mathrm{KWs}$ ).

- The KS and LN prefactors contain very different numerical constants $C$ : an order-one constant in $\mathrm{LN}$ $\left(C_{\mathrm{LN}} \sim 1\right.$, yet to be found) and a zero constant in $\mathrm{KS}$ ( $C_{\mathrm{KS}}=0$ as a formal consequence of its nonlocality). Also we should note a mysterious very small numerical factor $10^{-5}$ in formula (12) for the energy flux in Ref. [12], that has no physical justification. Actually, nonlocality of the energy transfer over scales means that this number should be very large, rather than very small. This emphasizes the confusion, and highlights the need for nu- merical re-evaluation of the spectrum's prefactor.

- Obviously, the differences between the KS and the LN spectra, in the exponents and, most importantly, in the prefactors, is important for practical analysis and interpretations of experimental data. At the same time, the difference between the underlying physics of the local and the nonlocal energy cascades, is important from the fundamental, theoretical viewpoint.

- In this work, the effective local 4-wave KE was derived from the 6 -wave $\mathrm{KE}$ by exploiting nonlocality of the latter which is valid only when the 6 -wave $\mathrm{KE}$ is valid, i.e. when all the scales are weakly nonlinear, including the ones at the IR cutoff. However, The resulting 4-wave $\mathrm{KE}$ is likely to be applicable more widely, when only the small scales, and not the large scales, are weak. A similar picture was previously observed for the nonlocal turbulence of Rossby/drift waves in [18] and for nonlocal MHD turbulence in [19]. In future we plan to attempt derivation of the 4 -wave $\mathrm{KE}$ directly from the dynamical equations for the KWs, which would allow us to extend its applicability to the case with strong large scales.

- Finally we note that the suggested here theory can potentially be useful for other one-dimensional physical systems, including optical fibers, where nonlinear interactions of one-dimensional wave packages becomes important with increase in network capacity.

Acknowledgements: We thank J. Laurie and O. Rudenko for help in the evaluation of the effective interaction coefficient. We acknowledge support of the US-Israel Binational Scientific Foundation administrated by the Israeli Academy of Science, and of the EC - Research Infrastructures under the FP7 Capacities Specific Programme, MICROKELVIN project number 228464.
[1] R. J. Donnelly, Quantized Vortices in He II (Cambridge University Press, Cambridge, 1991).

[2] W. F. Vinen and J. J. Niemela, J. Low Temp. Phys. 128, 167 (2002).

[3] Eltsov, V. B., Krusius, M. and Volovik, G. E. (2005). Vortex Formation and Dynamics in Superfluid $3 \mathrm{He}$ and Analogies in Quantum Field Theory, in edited by Halperin, W. P. (ed.), Progress Low Temperature Physics, vol. XV, p. 1, Elsevier B. V., Amsterdam.

[4] V.B. Eltsov et al., Phys. Rev. Letts, 99, 265301 (2007);

[5] V.B. Eltsov et al., Progress in Low Temp. Phys., XVI pp. 46-146 (2009)

[6] P.M. Walmsley, et al., Phys. Rev. Lett. 99, 265302 (2007).

[7] V. Bretin et al., Phys. Rev. Lett. 90, 100403 (2003).

[8] T. Mizushima et al., Phys. Rev. Lett. 90, 180401 (2003).

[9] W.F. Vinen, M. Tsubota and A. Mitani, Phys. Rev. Lett. 91, 135301 (2003).

[10] J. Yepezet al., Phys. Rev. Lett. 103, 084501 (2009).

[11] W. Thomson (Lord Kelvin), Philos. Mag. 10, 155 (1880).

[12] E. Kozik and B. Svistunov, Phys. Rev. Lett. 92, 035301
(2004); Phys. Rev. B 77, 060502(R) (2008); J. of Low Temp. Phys., 215, 156, (2009).

[13] J. Laurie, V. S. L'vov, S. V. Nazarenko and O. Rudenko, Phys. Rev. B. submitted, also arXiv:0911.1733.

[14] G. Boffetta, A. Celani, D. Dezzani, J. Laurie and S. Nazarenko, J. of Low Temp. Phys., 156, 193 (2009).

[15] V .E. Zakharov, V.S. L'vov and G.E. Falkovich. Kolmogorov Spectra of Turbulence, (Springer-Verlag, 1992).

[16] V. S. L'vov, S. V. Nazarenko, O. Rudenko, Phys. Rev. B 76, 024520 (2007); Journal of Low Temp. Phys., 153, 140-161 (2008).

[17] C. Connaughton, S. Nazarenko and A.C. Newell, Physica D vol. 184 (2003) pp. 86-97.

[18] S.V. Nazarenko, Ph.D. Thesis, Landau Inst. for Theor. Phys., Moscow, 1990.

[19] S. Galtier, S.V. Nazarenko and A.C. Newell, Physica D, 152-153 (2001) 646-652. 
Appendix: Proof of locality of the energy transfer in the $(1 \leftrightarrow 3)$-wave processes.

Mathematically, locality of the energy transfer in the $(\mathbf{1} \leftrightarrow \mathbf{3})$-wave processes means convergence of the multi-dimensional integral in the corresponding collision term (4b). Here we will show that proof of convergence in Eq. (4b) is a delicate issue and cannot be done only on the basis of power counting because the latter would give a divergent answer.

a. Proof of the infrared (IR) convergence. Let us show that in the IR region, when at least one of the wave vectors, say $k_{2}$, is much smaller then $k$, only a quadruple cancelation of the largest, next to the largest and the two further sub-leading contributions appear to result in the final, convergent result for the collision term (4b).

Three integrations in Eq. (4b) are restricted by two conservation laws, namely by

$$
1 \rightarrow 3: \quad \boldsymbol{k}=\boldsymbol{k}_{1}+\boldsymbol{k}_{2}+\boldsymbol{k}_{3}, k^{2}=k_{1}^{2}+k_{2}^{2}+k_{3}^{2} ;
$$

in the first term and by

$$
3 \rightarrow 1: \quad \boldsymbol{k}+\boldsymbol{k}_{2}+\boldsymbol{k}_{3}=\boldsymbol{k}_{1}, k^{2}+k_{2}^{2}+k_{3}^{2}=k_{1}^{2} ;
$$

in the second term. Therefore, only one integration, say with respect to $\boldsymbol{k}_{2}$, remains in each term.

In the IR region $k_{2} \ll k_{1} \ll k$, we find from Eq. (14) for the $(1 \rightarrow 3)$ and the $(3 \rightarrow 1)$ terms:

$$
\begin{aligned}
& 1 \rightarrow 3: \quad \boldsymbol{k}_{1}=\boldsymbol{k}-\frac{k_{2}^{2}}{\boldsymbol{k}_{1}+\boldsymbol{k}_{2}} \approx \boldsymbol{k}-\frac{\boldsymbol{k}_{2}^{2}}{\boldsymbol{k}}, \\
& 1 \rightarrow 3: \quad \boldsymbol{k}_{3}=-\frac{\boldsymbol{k}_{1} \boldsymbol{k}_{2}}{\boldsymbol{k}_{1}+\boldsymbol{k}_{2}} \approx-\boldsymbol{k}_{2}, \\
& 3 \rightarrow 1: \quad \boldsymbol{k}_{1}=\boldsymbol{k}+\frac{k_{2}^{2}}{\boldsymbol{k}+\boldsymbol{k}_{2}} \approx \boldsymbol{k}+\frac{k_{2}^{2}}{\boldsymbol{k}}, \\
& 3 \rightarrow 1: \quad \boldsymbol{k}_{3}=-\frac{\boldsymbol{k} \boldsymbol{k}_{2}}{\boldsymbol{k}+\boldsymbol{k}_{2}} \approx-\boldsymbol{k}_{2} .
\end{aligned}
$$

These equations demonstrate three important facts:

1. in both cases in the leading order $k_{3} \simeq k_{2}$, i.e. when $k_{2} \ll k$ then $k_{3}$ is small as well;

2. the difference between $\boldsymbol{k}_{1}$ and $\boldsymbol{k}$ is of the second order in small $k_{2}:\left|\boldsymbol{k}_{1}-\boldsymbol{k}\right| \simeq k_{2}^{2} / k$;

3. these leading contributions to $\left(\boldsymbol{k}_{1}-\boldsymbol{k}\right)$ have the same modulus and different sign in the $(1 \leftrightarrow 3)$ term and in the $(3 \leftrightarrow 1)$-term.

Therefore in the leading order the expressions for $\mathcal{N}$ in Eq. (4b) can be written as:

$$
\begin{aligned}
& \mathcal{N}_{k}^{1,2,3} \simeq-x\left(k_{2} / k\right)^{2} n_{k} n_{2} n_{3} \simeq-\frac{x A^{3}}{k^{(x+2)}} k_{2}^{2(1-x)}, \\
& \mathcal{N}_{1}^{k, 2,3} \simeq+x\left(k_{2} / k\right)^{2} n_{k} n_{2} n_{3} \simeq+\frac{x A^{3}}{k^{(x+2)}} k_{2}^{2(1-x)}
\end{aligned}
$$

where we substituted $n_{j}$ from Eq. (5). Importantly, these estimates (in the leading order) have the same magnitude and different signs.

Next step is to compute integrals

$$
\begin{aligned}
& I_{1 \rightarrow 3} \equiv \int d \boldsymbol{k}_{1} d \boldsymbol{k}_{3} \delta\left(\boldsymbol{k}-\boldsymbol{k}_{1}-\boldsymbol{k}_{2}-\boldsymbol{k}_{3}\right) \\
& \times \delta\left(k^{2}-k_{1}^{2}-k_{2}^{2}-k_{3}^{2}\right)=\frac{\left|\boldsymbol{k}+\boldsymbol{k}_{2}\right|}{2\left|k^{2}+2 \boldsymbol{k}_{2}-k_{2}^{2}\right|} \rightarrow \frac{1}{2 k} ; \\
& I_{3 \rightarrow 1} \equiv \int d \boldsymbol{k}_{1} d \boldsymbol{k}_{3} \delta\left(\boldsymbol{k}+\boldsymbol{k}_{2}+\boldsymbol{k}_{3}-\boldsymbol{k}_{1}\right) \\
& \times \delta\left(k^{2}+k_{2}^{2}+k_{3}^{2}-k_{1}^{2}\right)=\frac{1}{2\left|\boldsymbol{k}+\boldsymbol{k}_{1}\right|} \rightarrow \frac{1}{2 k},
\end{aligned}
$$

i.e. in the leading order these results coincide and do not contain the smallness.

Now we can find the contributions to $\mathrm{St}_{1 \leftrightarrow 3}$, given by Eq. (4b), from the region $k_{2} \ll k$. According to Eq. (11) we can write $V_{k}^{1,2,3}=V_{1}^{k, 2,3}=V \boldsymbol{k} \boldsymbol{k}_{1} \boldsymbol{k}_{2} \boldsymbol{k}_{3}$. Using our estimates (16) for $\mathcal{N}$ and Eq. (17) we have:

$$
\begin{aligned}
& 1 \rightarrow 3: \mathrm{St}_{1 \rightarrow 3}^{k_{2} \ll k} \approx-\frac{x \pi V^{2} A^{3}}{24 k^{x-1}} \int k_{2}^{2(3-x)} d \boldsymbol{k}_{2} ; \\
& 3 \rightarrow 1: \mathrm{St}_{1 \rightarrow 3}^{k_{2} \ll k} \approx+\frac{3 x \pi V^{2} A^{3}}{24 k^{x-1}} \int k_{2}^{2(3-x)} d \boldsymbol{k}_{2} .
\end{aligned}
$$

One can see that, in spite of the deep cancelations in the estimates for $\mathcal{N}$, the integrals (18) diverge if $x \geq 3.5$, which is satisfied for LN-scaling exponent $x=11 / 3$.

Nevertheless on has to take into account the following: the $(1 \rightarrow 3)$-contribution to the collision integral has three identical divergent regions: $k_{2} \sim k_{3} \ll k_{1} \approx k$, $k_{1} \sim k_{3} \ll k_{2} \approx k$ and $k_{2} \sim k_{1} \ll k_{3} \approx k$, and Eq. (18a) estimates only the first one. Therefore the total contribution is

$$
\mathrm{St}_{1 \rightarrow 3}^{\mathrm{IR}}=3 \mathrm{St}_{1 \rightarrow 3}^{k_{2} \ll k} \approx-\frac{3 x \pi V^{2} A^{3}}{24 k^{x-1}} \int k_{2}^{2(3-x)} d \boldsymbol{k}_{2},
$$

while the $(3 \rightarrow 1)$-contribution has only one divergent region $\boldsymbol{k}_{1} \approx \boldsymbol{k}$. Therefore,

$$
\mathrm{St}_{1 \rightarrow 3}^{\mathrm{IR}}=\mathrm{St}_{1 \rightarrow 3}^{k_{2} \ll k} \approx+\frac{3 x \pi V^{2} A^{3}}{24 k^{x-1}} \int k_{2}^{2(3-x)} d \boldsymbol{k}_{2},
$$

i.e. exactly the same result as in Eq. (18a), but with the different sign. Therefore the divergent contributions (18) cancel each other and one has to take into account the next order.

Notice that next order terms in the expansion over $k_{2} \ll k$ results in the already convergent integral

$$
\mathrm{St}_{1 \leftrightarrow 3}^{\mathrm{IR}} \propto \int_{0}^{k_{\mathrm{IR}} \ll k} \boldsymbol{k}_{2} k_{2}^{(6-2 x)} d \boldsymbol{k}_{2},
$$

with the LN exponent $x=11 / 3$. Moreover, typically excitation of KWs is symmetrical in $\boldsymbol{k} \leftrightarrow-\boldsymbol{k}$. In this 
case, this integral has an odd integrand and, therefore, it is equal to zero. Then the leading contribution to the $(1 \leftrightarrow 3)$-collision term in the IR region can be summarized as follows:

$$
\begin{gathered}
\mathrm{St}_{1 \leftrightarrow 3}^{\mathrm{IR}} \sim \frac{V^{2} A^{3}}{k^{x+1}} \int_{0}^{k_{\mathrm{IR}} \ll k} k_{2}^{2(4-x)} d \boldsymbol{k}_{2} \propto k_{\mathrm{IR}}^{9-2 x} . \\
\text { The IR convergence require: } \quad x<\frac{9}{2} .
\end{gathered}
$$

With LN exponent $x=\frac{9}{3}$ this gives $\mathrm{St}_{1 \leftrightarrow 3}^{\mathrm{IR}} \propto k_{\mathrm{IR}}^{5 / 3} \equiv$ $k_{\mathrm{IR}}^{\delta_{\mathrm{IR}}}$. Here we introduce an "IR convergence reserve": $\delta_{\mathrm{IR}}=\frac{5}{3}$.

b. Proof of the ultraviolet (UV) convergence. Convergence of the integral (4b) in the UV region, when one of the wave vectors, say $k_{2} \gg k$, can be established i a similar manner.

Notice first of all that in the $1 \rightarrow 3$ term in Eq. (4b), there is no UV region, because by the $2^{\text {nd }}$ of Eq. (14a) we have $k_{j} \leq k$. In the $3 \rightarrow 1$ term to satisfy Eq. (14b) in the leading order we can take $\boldsymbol{k}_{2} \simeq \boldsymbol{k}_{1} ; k_{2} \geq k_{\mathrm{UV}} \gg k$ (case $\boldsymbol{k}_{3} \simeq \boldsymbol{k}_{1} ; \quad k_{3} \geq k_{\mathrm{UV}}$ gives an identical result). Using parametrization $\boldsymbol{k}_{1}=\boldsymbol{k}+k_{2}^{2} /\left(\boldsymbol{k}+\boldsymbol{k}_{2}\right), \quad \boldsymbol{k}_{3}=$ $-\boldsymbol{k} \boldsymbol{k}_{2} /\left(\boldsymbol{k}+\boldsymbol{k}_{2}\right)$ (cf. (16b)) we get some cancelations in $\mathcal{N}_{1}^{k, 2,3}$ and the leading order result is

$$
\mathcal{N}_{1}^{k, 2,3} \propto x(x+1)\left(\frac{k_{2}}{k}\right)^{-2-x}-\left(\frac{k_{2}}{k}\right)^{-2 x} .
$$

Further, similarly to Eqs. (17), one gets $I_{3 \rightarrow 1} \simeq 1 / k_{2}$. As before, the interaction coefficient $V \propto k_{2}^{2}$ or $V^{2} \propto k_{2}^{4}$. Counting the powers of $k_{2}$ one gets:

$$
\mathrm{St}_{1 \leftrightarrow 3}^{\mathrm{UV}} \propto k_{\mathrm{UV}}^{y}, y=\max (-2 x+4,-x+2) .
$$

The UV convergence require $y<0 \Rightarrow x>2$. (22)

One concludes that in the case $x=\frac{11}{3}, \mathrm{St}_{1 \leftrightarrow 3}^{\mathrm{UV}} \propto k_{\mathrm{UV}}^{-5 / 3} \equiv$ $k_{\mathrm{UV}}^{-\delta_{\mathrm{UV}}}$, where we introduce an "UV convergence reserve" $\delta_{\mathrm{UV}}=\frac{5}{3}$.

Notably, $\delta_{\mathrm{IR}}=\delta_{\mathrm{UV}}$. This equality is not occasional. Observed "counterbalanced" IR-UV locality is a consequence of the scale-invariance of the problem. Indeed, for a given values of $k_{\mathrm{IR}} \ll \widetilde{k} \ll k_{\mathrm{UV}}$ the IR-energy flux $k_{\mathrm{IR}} \Rightarrow \widetilde{k}$ (from the IR region $k \leq k_{\mathrm{IR}}$ toward the region $\sim \widetilde{k})$ should scale with $\left(k_{\mathrm{IR}} / \widetilde{k}\right)$ exactly in the same manner as the UV-energy flux $\widetilde{k} \Rightarrow k_{\mathrm{UV}}$ (from the $\widetilde{k}$ region toward the UV-region $k \leq k_{\mathrm{UV}}$ ) scales with $\widetilde{k} / k_{\mathrm{UV}}$. This is because the UV-flux $\widetilde{k} \Rightarrow k_{\mathrm{UV}}$ from $\widetilde{k}$-region can be considered as the IR flux toward $k_{\mathrm{Uv}}$-region. Remembering that the IR-energy flux $k_{\mathrm{IR}} \Rightarrow \widetilde{k}$ scales like $\left(k_{\mathrm{IR}} / \widetilde{k}\right)^{\delta_{\mathrm{IR}}}$, while the UV-flux $\widetilde{k} \Rightarrow k_{\mathrm{UV}}$ is proportional to $\left(\widetilde{k} / k_{\mathrm{UV}}\right)^{\delta_{\mathrm{UV}}}$, one immediately concludes that $\delta_{\mathrm{IR}}$ should be equal to $\delta_{\mathrm{UV}}$.

The overall conclusion is that the collision term $\mathrm{St}_{1 \leftrightarrow 3}$ is convergent in both the IR and the UV regions for $x=$ $\frac{11}{3}$ and the energy transfer in the $1 \leftrightarrow 3$ kinetic equation is local. 